\title{
Regenerative Effects of Wharton's Jelly Stem Cells-Conditioned Medium in UVA-Irradiated Human Dermal Fibroblasts
}

\author{
Yohanes Widodo Wirohadidjojo, Arief Budiyanto, Hardyanto Soebono \\ Department of Dermato-Venereology, Faculty of Medicine, Gadjah Mada University, Sekip Utara, Yogyakarta, INDONESIA \\ *Email for Correspondence: widiokarsono@yahoo.com
}

\begin{abstract}
Background: Ultraviolet A radiation (UVA) can photo-age skin by suppressing the proliferation, migration, and collagen deposition of human dermal fibroblasts (HDFs). This process occurs because UVA light can inhibit the gene expression of the TGF- $\beta$ receptor in HDFs. Moreover, Wharton's Jelly Stem Cells-Conditioned Medium (WJSC-CM) is hypothesized to release microvesicles that contain short m-RNA with regenerative properties.

Objectives: This study aimed to determine the regenerative properties of WJSC-CM on UVA-Irradiated Human Dermal Fibroblasts (UVA-HDFs)

Methods: Passaged fourth of HDFs obtained from the foreskin of six (11- to 13-year-old) boys were repeatedly irradiated with a total of $10 \mathrm{~J} / \mathrm{cm}^{2} \mathrm{UVA}$ and treated with various concentrations of WJSC-CM. We used non-irradiated HDFs as positive control. After that, the consumption of TGF- $\beta$, cellular proliferation, cellular migration, and collagen deposition of each group were measured and compared.

Results: Compared to the non-irradiated groups, the proliferation rates, migration rates, and collagen deposition of UVA-HDFs significantly decreased $(p<0.05)$. WJSC-CM can improve the consumption of TGF- $\beta$, proliferation, and cellular migration of UVA-HDFs. However, WJSC-CM failed to improve the collagen deposition of UVA-HDFs ( $p>0.05$ ).

Conclusions: WJSC-CM has regenerative properties and is a candidate material for the treatment of prematurely ageing skin induced by UVA-irradiation.
\end{abstract}

Key words: collagen depositions, migration, photo-ageing fibroblasts, proliferations, TGF- $\beta$, UVA, Wharton's jelly stem cells conditioned-medium

Ethical permission: This experiment was permitted by the local ethical permission committee Ref:KE/FK/382/EC with permission letter dated 17-04-2013.

This article is is licensed under a Creative Commons Attribution-NonCommercial 4.0 International License.

Attribution-NonCommercial (CC BY-NC) license lets others remix, tweak, and build upon work non-commercially, and although the new works must also

acknowledge \& be non-commercial.

\section{INTRODUCTION}

Solar radiation, especially ultraviolet A light (UVA), can prematurely age skin. Various studies have shown that the collagen content of exposed human skin is lesser than that of unexposed skin (Yamauchi etal, 1991; Chung etal, 2001; Schwartz etal, 2008) based on the levels of procollagen-1 expression and procollagen-1 m-RNA (Chung etal, 2001). These phenomena may be caused by UVA light, which produces reactive oxygen species (ROS) that affect human dermal fibroblasts (HDFs) in several ways. Among HDFs, ROS induce DNA damage that prolongs G1 arrest for DNA repair (Pignolo etal, 1998; Auclair etal, 2010) and AP-1 expression that results in the gene expression of matrix metalloproteinase (MMP) to lead to collagen degradation (Wlaschek etal, 1995; Choi etal, 2007). Moreover, ROS induce AP-1 expression can also inhibit membrane TGF- $\beta 1$ receptor gene expression (Quan etal, 2001; Fisher etal, 2002; Quan etal, 2002; Quan etal, 2004; Quan etal, 2005). Also, degradable collagen materials may inhibit new collagen synthesis (Varani etal, 2006), and the decreasing dermal collagen contents may shrink HDFs and change their TGF- $\beta 1$ receptor structure to disrupt TGF- $\beta 1$ signalling (Quan etal, 2013). All of these processes impair the function of photo-aged HDFs. 
Various methods have been developed to treat wrinkles in prematurely aging skin, ranging from fillers of autologous young fibroblasts isolated from gingiva (Watson etal, 1999; Burgess, 2005) and Botox injection (Burgess, 2005) to the utilisation of secretory factors of adipose-derived stem cells (Kim etal, 2009). Conversely, Wharton's jelly from the umbilical cord has been considered a source of mesenchymal stem cells or WJSCs (Wang etal, 2004; Wu etal, 2007), and these stem cells have been proven to accelerate complete wound healing with the formation of the epithelium on the skin of a sheep's back (Azari etal, 2011). This happens because these cells have ability to differentiate into various cells, including differentiate into sweat gland-like cells which are important components of skin epithelial (Xu etal, 2012). Biancone et al. (2012), in a study supported by Bruno and Bussolati (2013), hypothesised that WJSCs may release microvesicles that contain short m-RNAs into their conditioned-medium, which can interfere with the local genetic programmes of cells cultured in this medium to induce cells to self-repair. Because the pathologic conditions of ageing fibroblasts in prematurely aging skin are similar to those of ageing fibroblasts in chronic ulcers (Watson and Griffiths, 2005), culturing UVA-irradiated HDFs (UVA-HDFs) in WJSCs conditioned-medium (WJSC-CM) may restore the activities of UVA-HDFs.

\section{Material AND Methods}

\section{Isolation and culture of HDFs}

We collected HDFs from the dermal part of the foreskins of six voluntary human subjects (11-13 years old) with informed consent. After mechanically removing of epidermal tissues from the skin, we cut the dermal parts into 2-4 $\mathrm{mm}^{3}$ pieces, placed in a culture flask, immersed in a small amount of growth medium (GM) consisting of high glucose Dulbecco's minimal essential medium (DMEM) (Gibco) supplemented with $10 \%$ foetal bovine serum (FBSGibco) and $1 \%$ penicillin/streptomycin (Gibco). Incubation was performed at $37^{\circ} \mathrm{C}$ until the tissues attached to the bottom of the flask. The medium was then exchanged with ten $\mathrm{mL}$ of fresh medium every 72 hours until $60 \%$ of the HDFs were outgrowing. The HDFs were then harvested and sub-cultured until passage $4^{\text {th }}$.

\section{Isolation and culture of WJSCS}

The isolation and culture of WJSCs were based on the explant technique of Ishige et al. (2009) with slight modifications. Briefly, 2-3 cm of umbilical cord obtained with informed consent from a full-term Caesarean section patient was rinsed with PBS three times for 10 minutes each, while blood was squeezed out and cord torsion was straightened. Subsequently, the cord was cut perpendicular to the long axis into 2-3 mm thick pieces. The pieces were then placed on the bottoms of 75 $\mathrm{cm}^{2}$ culture flasks that were already coated with $1 \mathrm{mg} / \mathrm{mL}$ type- 1 collagenase (Sigma) and incubated for 30 minutes at $37^{\circ} \mathrm{C}$. After the collagenase has been aspirated, the explants were carefully rinsed with PBS, immersed in $1 \mathrm{~mL}$ of low glucose DMEM with two ng/ mL b-FGF (Sigma), and incubated in $37^{\circ} \mathrm{C}$ with $5 \% \mathrm{CO}_{2}$. Medium replacement was done every day until explants attached to the flask's bottom. Furthermore, the medium was then replaced with growth medium that consisted of low glucose/Ham-F12 DMEM (Gibco) supplemented with $10 \%$ PBS, $2 \mathrm{ng} / \mathrm{mL}$ b-FGF, $2.5 \mu \mathrm{g} / \mathrm{mL}$ amphotericin B (Gibco), and $1 \%$ penicillin/streptomycin. This last step was repeated every 72 hours until $60 \%$ of fibroblast-like cells were observed to be outgrowing (fig.1A and B).

\section{Collection of WJSC-CM}

WJSC-CM was collected from the supernatant of spheroid-bodies of a high-density WJSCs culture according to a method published by Kurosawa (2007). Briefly, $7.5 \times 10^{5} / \mathrm{mL}$ passage-4 WJSCs were cultivated in growth medium in 24 well culture-plates until spheroid- bodies formed (fig.1C and D). At this condition, the growth medium was then replaced with low glucose/Ham-F12 DMEM containing $1 \%$ FBS for 72 hours as previously performed by Potapova etal. (2007). Subsequently, the supernatant then was collected, centrifuged at $200 \mathrm{~g}$ for 10 minutes, and maintained at $-20^{\circ} \mathrm{C}$ until use.

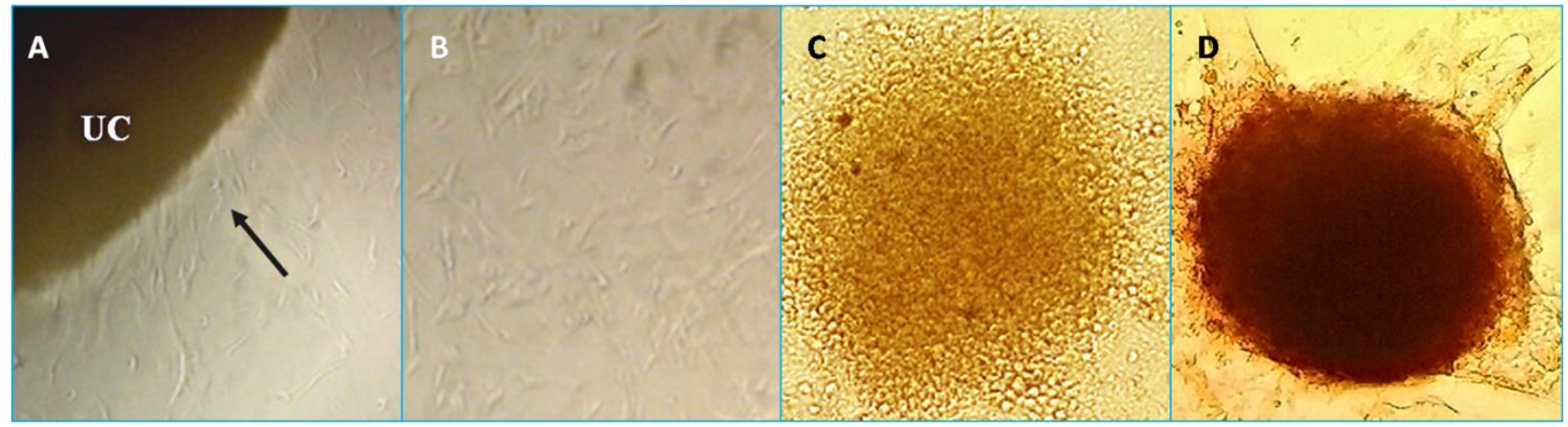

Figure 1: Isolation, cultivation, and spheroid body formation of WJSCs

A. An outgrowth of fibroblasts-like cells from explant, B. Culture of WJSCs, C. High- density cultivation of WJSCs, D. Spheroidbody of WJSCs 


\section{Experiments}

UVA-irradiated HDFs: UVA irradiations onto HDFs were prepared based on a procedure of UVA-induced aging of HDFs that published by Naru etal. (2005). Briefly, three replicates of $200 \mu \mathrm{L}$ of $2 \times 10^{3} / \mathrm{mL}$ passage-4 HDFs from different sources were cultured in each well of a 96-well plate in GM in $5 \% \mathrm{CO} 2$ at $37^{\circ} \mathrm{C}$ for 24 hours. The HDFs were then washed with PBS and exposed to UVA with 3-4 drops of PBS. UVA irradiation provided by PasSun A lamps (LIPI Indonesia) and the irradiance was determined using a photometer (Davlin X-96 detector-USA). The UVA irradiation dosage was $3.33 \mathrm{~J} / \mathrm{cm}^{2}$ for each radiation treatment. Immediately after the irradiation, the PBS was aspirated, replaced with complete medium, and the cells were returned to the incubator. Irradiation was repeated every 72 hours until the total dose approached $10 \mathrm{~J} / \mathrm{cm}^{2}$. Also, non-UVA-irradiated HDFs or normal fibroblasts were cultured in different plates with similar washing and medium replacement procedures.

Treatment with WJSC-CM: Immediately after the final UVA irradiation, UVA-irradiated HDFs were treated with 200 $\mu \mathrm{L}$ of $100 \%, 50 \%$, or $25 \%$ WJSC-CM diluted in DMEM supplemented with $1 \%$ FBS or control medium (CM) that consisted of DMEM plus $1 \%$ FBS. NFs were co-cultured in GM and CM. All cells were cultured for 72 hours in $37^{\circ} \mathrm{C}$ and $5 \% \mathrm{CO}_{2}$, and the medium was replaced once every 72 hours. Each treatment was replicated three times. The WJSC-CM concentration that resulted in the best proliferation rate was used in subsequent experiments to assess the migration ability, collagen deposition, and TGF- $\beta$ consumption of UVA-irradiated HDFs.

\section{Measurements}

Proliferation index: Cellular viability was measured using a MTT (-(3-(4,5-dimethylthiazol-2-yl)-2,5diphenyltetrazolium bromide) assay. The medium removed, the cells were washed with PBS, and $200 \mu \mathrm{L}$ complete medium plus $50 \mu \mathrm{L}$ of $(5 \mathrm{mg} / \mathrm{mL}$ ) MTT (MP biomedical-France) was then added to each well. The plates were then wrapped with aluminium foil and incubated at $37^{\circ} \mathrm{C}$ in $5 \% \mathrm{CO}_{2}$ for 4 hours. The medium and MTT then removed and the remaining MTT-formazan was then dissolved by adding $200 \mu \mathrm{L}$ DMSO (Dimethyl sulfoxide). The optical density resulting from the formazan in each well then was measured at $570 \mathrm{~nm}$ using a spectrophotometer.

$$
\text { Determination of proliferation rates were as }=\frac{\text { The OD of experimental groups }}{\text { The OD of Normal HDFS }} \times 100 \%
$$

Cellular migration assay: A cellular migration assay was performed based on a method byYarrow etal. (2004) and computed based on a method by Liang etal. (2007) with little modification. Briefly, after the final UVA irradiation, all wells were linearly scratched with the blunt tip of a $32 \mathrm{G}$ sterile needle through the centre of the well bottom. After treatment followed by 3 incubations of 72 hours each, the cells were stained with Meyer haematoxylin and microscopic photo images were taken using a Moticam-350 (China) camera in JPG format. The capacity for migration was determined via the computerised counting of blue fibroblast pixel-numbers compared to the non-blue pixel-numbers of the remaining free space along the scratching lines.

Cellular collagen deposition rate: The collagen deposition assay was based on an insoluble collagen of Sirius red assay according to Taskiran etal. (1999). Briefly, the wells were washed with PBS after treatment, and the cells were fixed with Boiun solution for one hour. The wells were then washed off with tap water and allowed to dry at room temperature overnight. Two hundred microliters of $0.1 \%$ Sirius red in saturated picric acid (SigmaAldrich, USA) were added to each well for one hour. The unbound Sirius red was washed away using four washes with $200 \mu \mathrm{l} 0.1 \mathrm{~N} \mathrm{HCl}$. The Sirius red bound to collagen was dissolved using $200 \mu \mathrm{L}$ of $0.5 \mathrm{~N} \mathrm{NaOH}$, and the optical density was read using a spectrometer at $570 \mathrm{~nm}$. Collagen deposition rates among experimental groups were calculated based on equation:

$$
\text { Collagen deposition was }=\frac{\text { OD of Experimental groups }}{\text { OD of Normal HDFs }} \times 100 \%
$$

TGF- $\beta$ Consumption: The levels of TGF- $\beta$ in the complete medium, $50 \%$ WJSC-CM, and cell culture supernatants were determined by direct ELISA using anti-human anti TGF- $\beta$ (Abcam cat.ab66043), HRP-conjugated secondary antibody (Abcam cat.ab6721), and 3,3,5,5,-tetra-methylbenzidine (Sure-BlueTMB microwell peroxidase substrate-KPL The USA). The optical densities were measured at $450 \mathrm{~nm}$ using a spectrophotometer. The optical densities were converted to TGF- $\beta$ concentrations based on an ELISA standard curve of serial dilutions of known levels of TGF- $\beta$ starting from $1000 \mathrm{pg} / \mathrm{mL}$.

$$
\text { Consumption of TGF- } \beta \text { was }=\frac{\text { Basic-level of TGF- } \beta \text { in medium }- \text { TGF- } \beta \text { supernatant of culture }}{\text { Basic-level of TGF- } \beta \text { in medium }} \times 100 \%
$$

\section{Statistics}

All the results are shown as an average \pm standard error. Comparisons among groups were analysed with an ANOVA test followed by LSD for a post hoc test. $\mathrm{P}<0.05$ was considered significant. 


\section{RESULTS}

The results can be observed in the following figures.
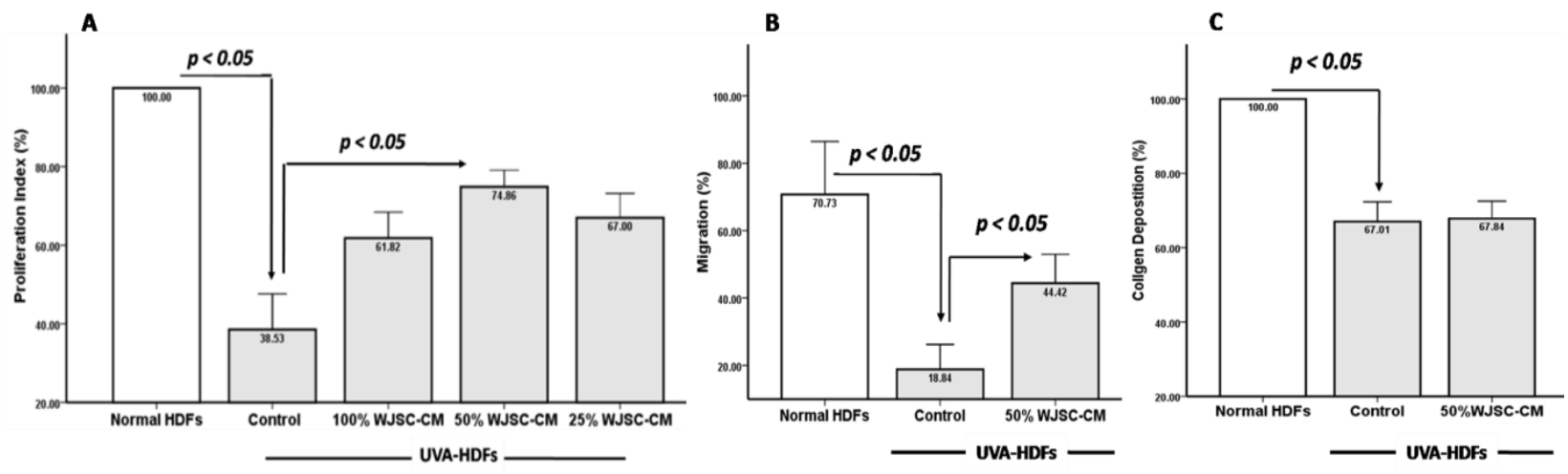

Figure 2: The effect of WJSCs-CM on activities of UVA-HDFs:

(A) on cellular proliferation, (B) on cellular migration, (C) on collagen deposition

On this figure, it can be observed that repeatly UVA-irradiation in total dosage of $10 \mathrm{KJ} . \mathrm{cm}^{-2}$ suppresses HDFs activities in cellular proliferation indexes, migration rates, and collagen deposition. Moreover, culture UVA-HDFs in $50 \%$ WJSC-CM can ameliorate their cellular activities in proliferation indexes and migration rates significantly $(p<0,05)$ but fail in collagen deposition activities. The change in TGF- $\beta$ receptor expressions of various groups in this experiment was measured by TGF- $\beta$ consumptions. On the following figure, they can be observed.

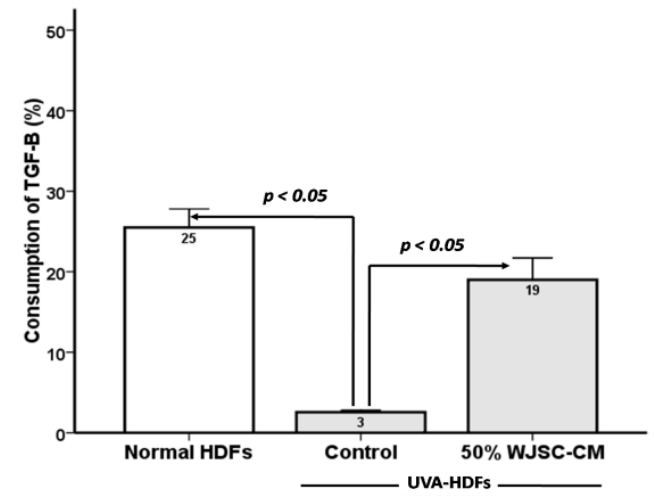

Figure 3: Consumption of TGF- $\beta$

On this figure, Consumption of TGF- $\beta$ among UVA-HDFs was significantly lower than normal HDFs $(p<0.05$ ) and cultured of UVA-HDFs in 50\% WJSC-CM had significantly higher. However, the improvement of TGF- $\beta$ consumption was still under normal HDFs's consumption ( $p>0.05$; data was not shown).

\section{Discussion}

Stem cells still express MHC on their membranes, which lead to a risk of donor rejection if they are used as transplanted foreign materials (Rossignol etal, 2009). Therefore, many researchers now focus on stem cells secretory factors for regenerative medicine. Various experiments have shown that factors secreted by stem cells into the conditioned medium have regenerative properties. For example, adipose-derived stem cells conditioned medium can induce proliferation, type I collagen synthesis, and inhibit MMP-1 expression in UVB-irradiated HDFs (Kim etal, 2009; Song etal, 2011; Kim etal, 2008). These effects may be associated to the antioxidant action of the conditioned medium of adipose-derived stem cells, as previously reported by Kim etal. (2008).

Similar to adipose-derived stem cells, WJSCs also release various secretory factors into their conditioned medium, and the most important secretory factors are microvesicles that contain short m-RNAs that may be transferred into injured cells, interfere with local genetic programming, and induce resident cells to re-enter the cell cycle, which leads cells to self-repair, as previously supposed by Biancone etal. (2012). This theory is based on the fact that WJSCs can reverse acute and chronic kidney injury in different experimental models via paracrine mechanisms (Bruno and Bussolati, 2013).

In the process of photo-aging skin, UVA light may generate ROS to prolong cell cycle G1 arrest for DNA repair DNA and stimulate AP-1 expression. AP-1 expression causes matrix metalloproteinase (MMP) gene expression, which leads to collagen degradation, and AP-1 expression blocks membrane TGF- $\beta 1$ receptor to inhibit the TGF- $\beta 1$ signalling. All of these behaviours may affect the proliferation, migration, and collagen deposition rates of UVA- 
HDFs, as shown in fig.2. In our experiments, the cultivation of UVA- HDFs in $100 \%, 50 \%$, and $25 \%$ WJSC-CM improved the cellular proliferation indexes compared with control as diluent media (fig.2). The cultivation of UVAHDFs in $50 \%$ WJSCM showed the best proliferation rates. Because the decreasing activities of UVA-HDFs are due to the impairment of the TGF- $\beta$ signalling pathway, these improved UVA-HDFs proliferation indexes might be caused by improved TGF- $\beta$ consumption, as demonstrated in fig.3. The improved TGF- $\beta$ signalling of UVA-HDFs might be caused by secretory factors that contain short-mRNAs released by WJSCs. These short-mRNAs might interfere with the genetic programming of UVA-HDFs to result in TGF- $\beta$ receptor gene expression self-repair, as indicated by TGF$\beta$ consumption. This improvement in the TGF- $\beta$ signalling of UVA-HDFs was also observed in the migration abilities of UVA-HDFs (fig.2). In this case, the improved UVA-HDFs migration rates were significantly higher than the migration abilities of normal-HDFs. The TGF- $\beta$-smad signalling pathway is well known to be important in the migration of HDFs (Montesano and Orci, 1988; Capelo, 2005; Hinz, 2007).

The collagen deposition assay showed an unexpected result (fig.2). Theoretically, improved TGF- $\beta$ signalling should restore the collagen deposition ability of UVA-HDFs because either TGF- $\beta$ signalling itself or its combination with other TGF- $\beta$ signalling pathway members induce connective tissue growth factor gene expression, which can stimulate collagen synthesis. Failure to stimulate collagen deposition by UVA-HDFs on above might be caused by the fact that WJSC-CM contains MMP-2 and MMP-9, as previously reported by Mauro et al. (2010). Both MMPs are gelatinases and can degrade gelatin as the by-product of collagen degradation induced by UVA radiation (Wlaschek etal, 1995; Naru etal, 2005). These gelatinase activities result in soluble materials that could not be detected by the Sirius red insoluble collagen assay. Also, degraded gelatin can stimulate the migration abilities of HDFs while inhibiting the ability of HDFs to synthesise new collagen (Xue etal, 2012).

Quan etal. (2013) reported that the improved TGF- $\beta$ signalling of dermal fibroblasts in an aged human skin is caused by the restoration the dermal microenvironment during skin aging. The increased collagen content in the microenvironment improves the mechanical force to elongate fibroblasts, couple with type I collagen synthesis, and upregulate TGF- $\beta$ receptor gene expression. Based on the lack of increased collagen deposition in fig.2, the improved TGF- $\beta$ signalling in UVA-HDFs shown in fig. 3 was not caused by improved microenviromental mechanical forces.

Sobolewski et al. (2005) reported that high-levels of TGF- $\beta$, b-FGF, EGF, PDGF, and IGF- 1 can be extracted from Wharton's jelly materials. The presence of these growth factors in WJSC-CM is still unclear, but WJSC-CM $50 \%$ in our experiments showed the presence of sufficient levels of TGF- $\beta$ (fig.3). Because PDGF is well known to be an important growth factor for wound healing (Werner and Grose, 2003) and injection of WJSC-CM can stimulate wound healing in mice (Zhang etal, 2012), PDGF likely plays a role in the regenerative properties of WJSC-CM. Also, various experiments showed that the PDGF signalling pathway can restore the TGF- $\beta$ signalling pathway in human dermal fibroblasts (Czuwara-Ladykowska etal, 2001) and mouse cardiac fibroblasts (Zhao etal, 2013). Therefore, the repair activities of UVA-HDFs cultured in WJSC-CM may be due to the high-level PDGF in WJSC-CM. Unfortunately, this assumption was not be evidenced in this experiment. We hope that this limitation study can be completed in further research.

\section{Conclusions}

Wharton's jelly stem cells-conditioned medium has regenerative properties that improve TGF- $\beta$ signaling, as well as cellular proliferation and migration rates of UVA-HDFs.

\section{REFERENCES}

Auclair Y, Rouget R, Belisle JM, Costantino S, Drobetsky EA. Requirement for functional DNA polymerase eta in genome-wide repair of UV-induced DNA damage during S phase., DNA Repair (Amst). 2010; 9(7):754-64.

Azari O, Babaei H, Derakhshanfar A, Nematollahi-Mahani SN, Poursahebi R, Moshrefi M. Effects of transplanted mesenchymal stem cells isolated from Wharton's jelly of caprine umbilical cord on cutaneous wound healing; histopathological evaluation. Vet Res Commun. 2011; 35(4):211-22.

Biancone L, Bruno S, Deregibus MC, Tetta C, Camussi G. Therapeutic potential of mesenchymal stem cell-derived microvesicles. Nephrol Dial Transplant. 2012; 27(8):3037-42.

Bruno S \& Bussolati B. Therapeutic effects of mesenchymal stem cells on renal ischemia-reperfusion injury: a matter of genetic transfer? Stem Cell Res Ther. 2013;4(5):55.

Burgess CM. Soft Tissues Augmentation., in Burgess CM(ed) : Cosmetic Dermatology., Springer., Washington DC. 2005; $93-110$.

Capelo AS. Dual role for TGF- $\beta 1$ in apoptosis., Cytokine E Growth Factor Reviews. 2005; 16(1): 15-34.

Choi CP, Kim YI, Lee JW, Lee MH. The effect of narrowband ultraviolet B on the expression of matrix metalloproteinase-1, transforming growth factor-beta1 and type I collagen in human skin fibroblasts., ClinExpDermatol. 2007; 32(2):180-5.

Chung JH, Seo JY, Choi HR, Lee MK, Youn CS, Rhie G, Cho KH, Kim KH, Park KC, Eun HC. Modulation of skin collagen metabolism in aged and photoaged human skin in vivo. J Invest Dermatol. 2001;117(5):1218-24.

Czuwara-Ladykowska J, Gore EA, Shegogue DA, Smith EA, Trojanowska M. Differential regulation of transforming growth factor-beta receptors type I and II by platelet-derived growth factor in human dermal fibroblasts. Br J Dermatol. 2001;145(4):569-75. 
Fisher GJ, Kang S, Varani J et al. Mechanisms of photoaging and chronological skin aging. Arch Dermatol. 2002; 138:1462-70.

Hinz B. Formation and function of the myofibroblast during tissue repair. J Invest Dermatol.2007; 127(3):526-537.

Ishige I., Nagamura-Inoue T., Honda MJ., HarnprasopwatR.,Kido M. et al.. Comparison of mesenchymal stem cells derived from arterial, venous, and Wharton's jelly explants of human umbilical cord., Int.J.Hematol. 2009; 90:261-69.

Kim WS, Park BS, Kim HK, Park JS, Kim KJ, Choi JS, Chung SJ, Kim DD, Sung JH. Evidence supporting antioxidant action of adiposederived stem cells: protection of human dermal fibroblasts from oxidative stress. J Dermatol Sci. 2008; 49(2):133-42

Kim WS, Park BS, Park SH, Kim HK, Sung JH. Antiwrinkle effect of adipose-derived stem cell: activation of dermal fibroblast by secretory factors. J Dermatol Sci. 2009;53(2):96-102.

Kurosawa H. Method For Inducing Embryoid Body Formation : Invitro Differentiation System of Embryonic Stem Cells , J.Biosci.Bioeng 2007; (103)5: 389-398.

Liang CC, Park AY, Guan JL. In vitro scratch assay: a convenient and inexpensive method for analysis of cell migration in vitro. Nat Protoc. 2007; 2(2):329-33.

Mauro A, Buscemi M, Gerbino A. Immunohistochemical and transcriptional expression of matrix metalloproteinases in full-term human umbilical cord and human umbilical vein endothelial cells., J Mol Histol. 2010;41(6):367-77.

Montesano R, Orci L. Transforming growth factor beta stimulates collagen-matrix contraction by fibroblasts: implications for wound healing. ProcNatlAcadSci USA, 1988; 85(13):4894-4897

Naru E, Suzuki T, Moriyama M, Inomata K, Hayashi A, Arakane K, Kaji K . Functional changes induced by chronic UVA irradiation to cultured human dermal fibroblasts., Br J Dermatol. 2005; 153 Suppl 2:6-12.

Pignolo RJ, Martin BG, Horton JH, Kalbach AN, Cristofalo VJ. The pathway of cell senescence: WI-38 cells arrest in late G1 and are unable to traverse the cell cycle from a true G0 state., ExpGerontol. 1998;33(1-2):67-80.

Potapova IA., Gaudette GR., Brink PR., et al. Mesenchymal Stem Cells Support Migration, Extracellular Matrix Invasion, Proliferation, and Survival of Endothelial Cells In Vitro., Stem Cells. 2007; 25:1761-1768.

Quan T, He T, Kang S, Voorhees J, Fisher G. Ultraviolet irradiation alters transforming growth factor $\beta /$ Smad pathway in human skin in vivo. J Invest Dermatol. 2002;119:499-506.

Quan T, He T, Kang S, Voorhees JJ, Fisher GJ. Solar ultraviolet irradiation reduces collagen in photoaged human skin by blocking transforming growth factor-beta type II receptor/Smad signaling. Am J Pathol. 2004; 165:741-751.

Quan T, He T, Voorhees J, Fisher G. Ultraviolet irradiation blocks cellular responses to transforming growth factor- $\beta$ by down-regulating its type-II receptor and inducing Smad7. J Biol Chem. 2001;276:26349-26356

Quan T, He T, Voorhees JJ, Fisher GJ.. Ultraviolet irradiation induces Smad7 via induction of transcription factor AP-1 in human skin fibroblasts. J Biol Chem. 2005; 280:8079-8085.

Quan T, Wang F, Shao Y, Rittié L, Xia W, Orringer JS, Voorhees JJ, Fisher GJ. Enhancing structural support of the dermal microenvironment activates fibroblasts, endothelial cells, and keratinocytes in aged human skin in vivo. J Invest Dermatol. 2013; 133(3):658-67

Rossignol J, Boyer C, Thinard R, Remy S, Dugast AS, Dubayle D, Dey ND, Boeffard F, Delecrin J, Heymann D, Vanhove B, Anegon I, Naveilhan P, Dunbar GL, Lescaudron L. Mesenchymal stem cells induce a weak immune response in the rat striatum after allo or xenotransplantation., J Cell Mol Med. , 2009; 13(8B):2547-58.

Schwartz E., Cruickshank FA., Christensen CC., Perlish JS., Lebwohl M. Collagen alteration in chronically sun damage human skin., Photochem.Photobiol.2008; 58:841-844

Sobolewski K., Malkowski A., Kowski EB., Jaworski S., Wharton's Jelly as a Reservoir of Peptide Growth Factors., Placenta.2005; 26:747-752

Song SY, Jung JE, Jeon YR, Tark KC, Lew DH. Determination of adipose-derived stem cell application on photo-aged fibroblasts, based on paracrine function., Cytotherapy. 2011; 13(3): 378-384

Taskiran D., Taskiran E., Yercan H., Kutay FZ. Quantification of total collagen in rabit tendon by the Sirius Red Methods, Tr J Med Science. 1999; (29):7-9.

Varani J,DameMK,Rittie L, FligielSEG,SewonKang,Fisher GJ, and Voorhees JJ. Decreased Collagen Production in Chronologically Aged Skin. Roles of Age-Dependent Alteration in Fibroblast Function and Defective Mechanical Stimulation., AJP. 2006;168 ( 6):1861-68

Wang HS, Hung SC, Peng ST, et al.. Mesenchymal stem cells in the Wharton's jelly of the human umbilical cord. Stem Cells. 2004; 22:1330-7

Watson D., Keller GS., Lacombe V., Fodor PB., Rawnsley J., Lask GP. Autologous Fibroblasts For Treatment of FascialRhytid and Dermal depressions., Arch.Fas.Plast.Surg.1999; 1: 165-170.

Watson RE, Griffiths CE. Pathogenic aspects of cutaneous photoaging. J CosmetDermatol 2005;4:230-6.

Werner S., Grose R., Regulation of Wound Healing by Growth Factors and Cytokines. Physiol Rev., 2003;83(3): 835-870.

Wlaschek M, Briviba K, Stricklin GP, Sies H, Scharffetter-Kochanek K., Singlet oxygen may mediate the ultraviolet A-induced synthesis of interstitial collagenase., J Invest Dermatol. 1995; 104(2):194-8.

Wu KH., Yang SG., Zou B., et al. Human umbilical cord derived stem cells for the injured heart., Med Hypo.2007; 68, 94-97.

Xu Y, Huang S, Ma K, Fu X, Han W, Sheng Z. Promising new potential for mesenchymal stem cells derived from human umbilical cord Wharton's jelly: sweat gland cell-like differentiative capacity., J Tissue EngRegen Med. 2012; 6(8):645-54.

Xue SN, Lei J, Yang C, Lin DZ, Yan L. The biological behaviors of rat dermal fibroblasts can be inhibited by high levels of MMP9. Exp Diabetes Res. 2012; 494-579.

Yamauchi M, Prisayanh P, Haque Z, Woodley DT. Collagen cross-linking in sun-exposed and unexposed sites of aged human skin., J Invest Dermatol. 1991; 97(5):938-41.

Yarrow J.C., Perlman Z.E., Westwood N.J., Mitchison T.J. A high-throughput cell migration assay using scratch wound healing, a comparison of image-based readout methods. BMC Biotechnol. 2004; 4: 21.

Zhang Y, Hao H, Liu J, Fu X, Han W. Repair and regeneration of skin injury by transplanting microparticles mixed with Wharton's jelly and MSCs from the human umbilical cord., Int J Low Extrem Wounds. ,2012;11(4):264-70

Zhao T, Zhao W, Chen Y, Li VS, Meng W, Sun Y. Platelet-derived growth factor promotes fibrogenesis of cardiac fibroblasts., Am J Physiol Heart Circ Physiol. 2013;304(12):H1719-26.

$$
-0 \text {-- }
$$

